\title{
Monte Carlo radiation transfer in CV disk winds: application to the AM CVn prototype
}

\author{
D.-J. Kusterer ${ }^{1}$, T. Nagel ${ }^{1}$, S. Hartmann ${ }^{1}$, K. Werner ${ }^{1}$, and A. Feldmeier ${ }^{2}$ \\ ${ }^{1}$ Institut für Astronomie und Astrophysik, Kepler Center for Astro and Particle Physics, Eberhard Karls Universität Tübingen, Sand 1, \\ 72076 Tübingen, Germany \\ e-mail: [nagel; werner] aastro.tuebingen.de \\ ${ }^{2}$ Institut für Physik und Astronomie, Universität Potsdam, Karl-Liebknecht-Str. 24/25, 14476 Potsdam, Germany \\ e-mail: afeld@astro.physik.uni-potsdam.de
}

Received 8 March 2013 / Accepted 10 November 2013

\begin{abstract}
Context. AMCVn systems are ultracompact binaries in which a (semi-) degenerate star transfers helium-dominated matter onto a white dwarf. They are effective gravitational-wave emitters and potential progenitors of Type Ia supernovae.

Aims. To understand the evolution of AMCVn systems it is necessary to determine their mass-loss rate through their radiationdriven accretion-disk wind. We constructed models to perform quantitative spectroscopy of P Cygni line profiles that were detected in UV spectra.

Methods. We performed 2.5D Monte Carlo radiative transfer calculations in hydrodynamic wind structures by making use of realistic NLTE spectra from the accretion disk and by accounting for the white dwarf as an additional photon source.

Results. We present first results from calculations in which LTE opacities are used in the wind model. A comparison with UV spectroscopy of the AMCVn prototype shows that the modeling procedure is potentially a good tool for determining mass-loss rates and abundances of trace metals in the helium-rich wind.
\end{abstract}

Key words. radiative transfer - stars: winds, outflows - stars: individual: AM CVn - accretion, accretion disks

\section{Introduction}

Accretion is a very common and important process in the Universe from powerful active galactic nuclei to young stellar objects. Yet many aspects of accretion and the physics of the accretion disks involved in the process are not fully understood. A prime example is the wind driven off a luminous accretion disk. Clear signatures of its presence are seen in spectral observations, but neither the driving mechanism nor the resulting mass-loss rates are determined in a satisfying manner. Ideal objects for a closer analysis of the accretion phenomenon are cataclysmic variable $(\mathrm{CV})$ stars. CVs are semi-detached binary systems with mass overflow from a late-type companion onto a white dwarf (WD) primary. Observational evidence for the presence of accretion disks and outflows in such systems has been known for a long time (Heap et al. 1978; Cordova \& Mason 1982). During the 1990s two kinematical models of an accretion-disk outflow that incorporate rotation were developed by Shlosman \& Vitello (1993, hereafter SV), and Knigge et al. (1995, hereafter KWD). Both models had their theoretical shortcomings, but were successfully used to reproduce observed spectra. At the turn of the century the theoretical background of accretion-disk winds was addressed in a series of papers (Feldmeier \& Shlosman 1999; Feldmeier et al. 1999; Proga et al. 1998, 1999). We return to these hydrodynamic backgrounds in Sect. 3 .

Long \& Knigge (2002) used a hybrid Sobolev/Monte Carlo method in combination with a self-consistent solution of the thermal and ionization balance as well as an approximation for non-local thermodynamic equilibirium (NLTE). They successfully reproduced far-ultraviolet (FUV) spectra of Z Cam. Based on this work, Noebauer et al. (2010) investigated FUV spectra of RW Tri and UX UMa. Compared with these works, our approach is simpler in that we assume a constant wind temperature as a given parameter as well as pure LTE. Recently, Puebla et al. (2011) investigated accretion-disk emission in CVs, calculating 1D-wind models in the comoving frame and combining these into 2.5D models for which they computed synthetic spectra. In contrast to their work, our wind models are calculated in 2.5 dimensions.

Not only in the usual CVs, but also in the ultracompact AM CVn systems, signatures of biconical accretion-disk winds were detected. The class of AMCVn stars is a CV subgroup whose secondary is believed to be a semi-degenerate low-mass star or WD transferring almost pure helium material to the primary (see, e.g., Solheim 2010, for a recent review). As in other CVs, an accretion-disk wind is mainly found in high-state AMCVn systems, a complete spectral model of such systems therefore has to include mass outflow. It is not clear a priori whether the models successfully used for standard CV systems and outflows are also valid for AMCVn systems. In this paper we show that these standard models also lead to a proper description of AMCVn systems and that therefore the driving mechanism behind outflows from hydrogen-rich and helium-rich outflows must be the same.

This paper is organized as follows: in Sects. 2 and 3 we introduce our kinematical and hydrodynamic wind models. In Sect. 4 we describe our Monte Carlo radiation transfer, followed by an introduction to our spectrum-modeling code for accretion disks (Sect. 5). Spectral properties of selected hydrodynamic and kinematical models are compared in Sect. 6. We then compare some of our models with ultraviolet spectra of the AM CVn prototype (Sect. 7) and conclude in Sect. 8. 


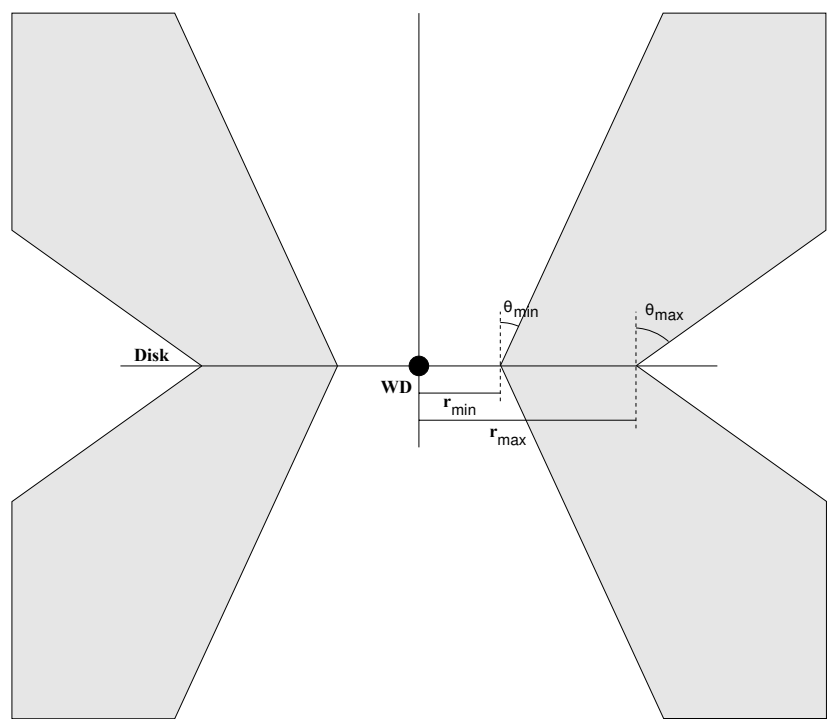

Fig. 1. Biconical structure and geometric parameters of the SV diskwind model.

\section{Kinematical wind model}

The first wind model for CVs that did not assume spherical symmetry was introduced by SV in 1993. They described such a wind in a straightforward way. The geometrical layout of their wind is shown in Fig. 1.

A biconical outflow without clumping or other effects such as a hot spot on the disk can be described by assuming axial symmetry. With this assumption a cylindrical set of coordinates is the natural choice to describe the system. The $z$ axis is taken to be co-aligned with the rotation axis of the disk. The $r$ and $\phi$ coordinates are the radial and azimuthal coordinates on the disk surface. We assumed a geometrically thin accretion disk, neglecting its $z$ extension. The wind is launched from the disk in an area between $r_{\min }$ and $r_{\max }$ and is described by intersectionfree streamlines. These streamlines are 3D helices lying on cones with constant opening angles $\theta$. The opening angle of the cones depends on the initial radius where the streamline is launched from

$\theta=\theta_{\min }+\left(\theta_{\max }-\theta_{\min }\right) x^{\gamma}$.

Here $x=\left(r_{0}-r_{\min }\right) /\left(r_{\max }-r_{\min }\right)$ and $r_{0}$ is the radial coordinate of the launching point on the disk surface. SV generally used $\gamma=1$, which corresponds to a linear variation of the opening angle. Here the same value for $\gamma$ is used unless specified otherwise. The limiting angles of the biconical wind structure are $\theta_{\min }$ and $\theta_{\max }$, which correspond to the cone angles at the inner and outer boundaries $r_{\min }$ and $r_{\max }$ of the wind.

With the chosen cylindrical coordinate system, streamline velocities in the wind are given in the components $v_{r}, v_{\phi}$ and $v_{z}$. The velocity along a streamline is denoted by $v_{1}$. Then simple geometry gives the relations $v_{r}=v_{1} \sin \theta$ and $v_{z}=v_{1} \cos \theta$. $\mathrm{SV}$ assumed that $v_{1}$ is given by a power law of the length $l=\left[\left(r-r_{0}\right)^{2}+z^{2}\right]^{1 / 2}$ along the streamline

$v_{1}=v_{0}+\left(v_{\infty}-v_{0}\right)\left[\frac{\left(l / R_{v}\right)^{\alpha}}{\left(l / R_{v}\right)^{\alpha}+1}\right]$,

where the initial and asymptotic wind velocities along the streamline $v_{0}$ and $v_{\infty}$ are taken as parameters of the model. The two other parameters in this velocity law are the acceleration scale height of the wind $R_{v}$ and $\alpha$ the power-law constant. In their model SV scaled the asymptotic velocity with local escape velocity at the streamline base, namely $v_{\mathrm{esc}}=\left(2 G M_{\mathrm{WD}} / r_{0}\right)^{1 / 2}$, with $M_{\mathrm{WD}}$ being the mass of the central WD and $G$ the gravitational constant. As initial velocity they assumed $v_{0}=6 \mathrm{~km} \mathrm{~s}^{-1}$. The wind in the streamlines not only has a velocity in $l$ direction, but also an orbital velocity $v_{\phi}$. It is initially given by the Keplerian motion of the disk at the streamline launching point $v_{\phi, 0}=\left(G M_{\mathrm{WD}} / r_{0}\right)^{1 / 2}$ and is only changed by the assumption of angular momentum conservation around the rotation axis, leading to

$v_{\phi} r=v_{\phi, 0} r_{0}$.

Shlosman \& Vitello (1993) took the mass-loss rate per unit surface of the disk in the direction of a streamline, $\dot{m}$, at the base radius $r_{0}$ to be a fraction of the total mass-loss rate by the wind $\dot{M}_{\text {wind }}$ given by

$\dot{m}\left(r_{0}\right)=\dot{M}_{\text {wind }} \frac{r_{0}^{\lambda} \cos \theta\left(r_{0}\right)}{\int \mathrm{d} A r_{0}^{\prime \lambda} \cos \theta\left(r_{0}^{\prime}\right)}$,

with the area integral being over the disk surface between $r_{\text {min }}$ and $r_{\max }$, or more explicitly,

$\int \mathrm{d} A=2 \int_{r_{\min }}^{r_{\max }} \mathrm{d} r r \int_{0}^{2 \pi} \mathrm{d} \phi$,

where the factor of two accounts for the lower and upper surface of the disk. Although one can accommodate a more complicated form of mass loss with this formula, SV generally used $\lambda=0$, which corresponds to a uniform mass loss. As the streamlines connect to the disk at an angle, the $\cos \theta$ factor is needed to correct for this.

Shlosman \& Vitello (1993) used an unequally spaced grid in $r, z$, and $\theta$ above and below the disk. Densities are calculated for each grid cell at the cell center. The density in the first cell above the disk is given by

$\rho\left(r_{0}\right)=\frac{\dot{m}\left(r_{0}\right)}{v_{z}\left(r_{0}\right)}$.

For points farther out in the wind the values of $r_{0}$ and $\theta\left(r_{0}\right)$ need to be determined first. Simple geometrical considerations, namely the projection of the $\theta$-run of the streamlines from a height $z$ to the disk at $z=0$, lead to

$r_{0}=r-z \tan \theta\left(r_{0}\right)$,

with

$\theta\left(r_{0}\right)=\theta_{\min }+\left(\theta_{\max }-\theta_{\min }\right) \frac{r-r_{\min , z}}{r_{\max , z}-r_{\min , z}}$,

where $r_{\text {min }, z}=r_{\text {min }}+z \tan \theta_{\min }$ and $r_{\max , z}=r_{\max }+z \tan \theta_{\max }$ are the $r$ values that limit the wind cone at height $z$. Then, with given $(r, z), r_{0}$ and $\theta\left(r_{0}\right)$, the velocity $v(r, z)=\left(v_{r}(r, z), v_{\phi}(r, z), v_{z}(r, z)\right)$ is readily calculated using Eqs. (2) and (3). The density at $(r, z)$ can then be calculated from the relation

$\rho(r, z)=\frac{r_{0}}{r} \frac{\mathrm{d} r_{0}}{\mathrm{~d} r} \frac{\dot{m}\left(r_{0}\right)}{v_{z}(r, z)}$.

As the wind flows away from the disk, the area between the streamlines increases. This is incorporated in the above equation via the $\left(r_{0} / r\right)\left(\mathrm{d} r_{0} / \mathrm{d} r\right)$ term, which is just the area between two streamlines at some height above the disk divided by the area 
between these two streamlines on the disk. Taking a geometrical view and keeping in mind that the distance $l$ along a streamline is $l(r)=z / \cos \theta(r)$, one can derive

$\frac{\mathrm{d} r}{\mathrm{~d} r_{0}}=1+\frac{z}{\cos ^{2} \theta(r)} \frac{\mathrm{d} \theta}{\mathrm{d} r_{0}}$.

Equipped with these densities and velocities, the wind structure can be calculated easily.

Additional assumptions of SV are a constant wind temperature of $20000 \mathrm{~K}$ and local ionization equilibrium. They considered $\mathrm{H}, \mathrm{He}, \mathrm{C}, \mathrm{N}$, and $\mathrm{O}$ with solar abundances, and solved the radiation transfer in Sobolev approximation. They included a boundary layer as radiation source in their model.

\section{Hydrodynamic model for CV winds}

Hydrodynamic models of disk winds driven by the radiation force resulting from photon scattering in numerous spectral lines (so-called line driving) have only recently been developed (Feldmeier \& Shlosman 1999; Feldmeier et al. 1999; Proga et al. 1998, 1999). The low wind mass-loss rates obtained in these papers revealed that fundamental processes are missing in the treatment. Most likely, these shortcomings stem from in the description of the line-driving force adopted from the CAK theory (Castor et al. 1975) for O star winds, which seems far too simplified for $\mathrm{CV}$ winds. The major shortcomings of that theory seem to be the assumptions of no line overlap (induced by Doppler-velocity shifts in the wind) and the highly simplified, two-parameter description of the complex NLTE situation of a relatively thin wind exposed to a strong UV radiation field. At present, we can only adopt results from the hydrodynamic models that agree with reliable empirical results on $\mathrm{CV}$ winds, most notably (i) that the wind originates from the inner regions of the accretion disk and is strongly biconical; and (ii) that the calculated wind mass-loss rate is on the order of the single-scattering limit. The role of magnetic torques and forces and of X-ray disk irradiation has to be clarified in future work.

The hydrodynamic models assuming a CAK line force have three unique features. (i) They show a critical point above the disk along a streamline where the flow speed equals that of radiative-acoustic waves (Abbott 1980); (ii) the Euler equation can be put in a form that reflects that of the Laval nozzle flow; (iii) both the mass-loss rate from a narrow disk ring and the inclination of the wind streamline with the disk normal are then uniquely fixed at the location where this nozzle function has its minimum.

The toroidal wind speed is fixed by assuming angular momentum conservation above a Keplerian disk. For the poloidal wind speed, we assumed that the parcel streamline projected onto the poloidal plane can be approximated by a straight line. Whereas the tilt of this wind cone with the disk normal is a fit parameter in the kinematical disk wind models by SV and KWD, it is, according to the above, a fixed ("eigen-") value in the dynamical model by Feldmeier \& Shlosman (1999). Comparing the disk wind structure resulting from hydrodynamic models and the structure assumed in the kinematical models, we see a strong similarity. Using hydrodynamic models, it is therefore possible to fix the free parameters of the kinematical models. Similarly, the radial dependence of the mass-loss rate per disk ring of infinitesimal extent $\mathrm{d} r$ (as well as the inner and outer termination radius of the wind) is parameterized in the kinematical models, and is uniquely determined in the dynamical model.

Since the integrated mass-loss rate from the hydrodynamic models is certainly too low, we scaled the latter upwards by an appropriate factor.

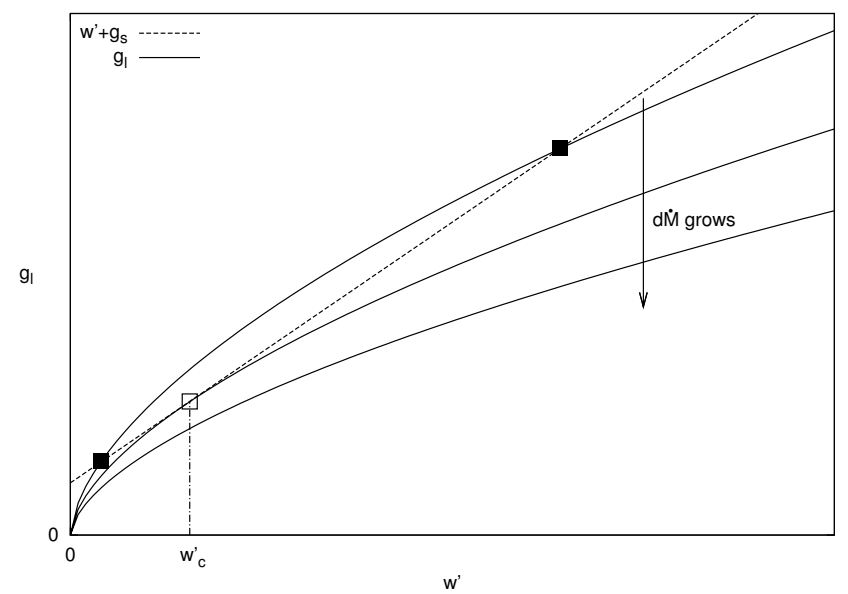

Fig. 2. Solution "topology" of the Euler equation, adopted from Cassinelli (1979). $w^{\prime}$ and $g_{s}$ are the two retarding forces, plotted positive, whose sum crosses the driving force $g_{1}$ at either zero, one (open square) or two points (closed squares). The line force is plotted for three values of the mass-loss rate. The critical point $w_{\mathrm{c}}^{\prime}$ is marked.

With the mass-loss rate leaving the disk in a ring of width $\mathrm{d} R$ at location $R$,

$\mathrm{d} \dot{M}_{\text {wind }}=2 \pi R \mathrm{~d} R \rho v \cos \theta$,

we follow Feldmeier \& Shlosman (1999) to find the Euler equation

$w^{\prime}+g_{s}-\eta \frac{n^{\alpha}}{\mathrm{d} \dot{M}_{\text {wind }}^{\alpha}} w^{\prime \alpha} g_{s}^{1-\alpha}=0$

with the nozzle function

$n=\frac{1}{\eta^{1 / \alpha}} 2 \pi R \mathrm{~d} R \cos \theta \frac{\left(C F_{s}\right)^{1 / \alpha}}{g_{s}^{(1-\alpha) / \alpha}}$.

Here $F_{s}$ is the flux projected onto the streamline $s$ and $C$ is a constant. The parameter $\eta$ was introduced to allow for a general value of $\alpha$.

The solution "topology" of Eq. (12) can be understood from Fig. 2 (adopted from Fig. 3 in Cassinelli 1979), which shows the line force $g_{1}$ as function of the inertia $w^{\prime}$ for three selected values of the mass-loss rate. These curves are concave from below since $\alpha<1$. This driving force is in balance with the two retarding forces (plotted positive in the figure), $w^{\prime}$ and the projected gravity $g_{s}$. Note that the inertial force is a $45^{\circ}$ diagonal in the diagram, and that $g_{s}$, depending only on $z$ not $w^{\prime}$, is a constant since a fixed yet arbitrary location is considered. The retarding and driving forces cross at either zero, one (open square in the diagram), or two points (closed squares). The latter two correspond to the so-called shallow and steep solutions. If the mass-loss rate adopts the critical CAK value, the shallow and steep solution merge into one. For still higher mass-loss rates, only imaginary solutions are possible, and the wind is called overloaded.

At the critical point (subscript c), one therefore has

$\frac{\partial g_{1}}{\partial w_{\mathrm{c}}^{\prime}}=1=\frac{\partial}{\partial w_{\mathrm{c}}^{\prime}}\left(w^{\prime}+g_{s}\right)$

or

$\frac{\partial}{\partial w_{\mathrm{c}}^{\prime}}\left(w^{\prime}+g_{s}-g_{1}\right)=0$.

This is the famous CAK critical-point condition. Note that the Euler equation is $w^{\prime}+g_{s}-g_{1}=0$, at every point, hence also at 
the critical point. This set of two equations (at the critical point) is readily solved, giving the well-known CAK relations

$w_{\mathrm{c}}^{\prime}=\frac{\alpha}{1-\alpha} g_{\mathrm{s}, \mathrm{c}}$

and

$\eta\left(\frac{n_{\mathrm{c}}}{\mathrm{d} \dot{M}}\right)^{\alpha}=\frac{1}{\alpha^{\alpha}(1-\alpha)^{1-\alpha}}$

Requiring $n_{\mathrm{c}}=\mathrm{d} \dot{M}_{\text {wind }}$, we arrive at

$\eta=\frac{1}{\alpha^{\alpha}(1-\alpha)^{1-\alpha}}$

thus the nozzle function is

$n=\alpha(1-\alpha)^{(1-\alpha) / \alpha} 2 \pi R \mathrm{~d} R \cos \theta \frac{\left(C F_{s}\right)^{1 / \alpha}}{g_{s}^{(1-\alpha) / \alpha}}$.

This indeed reproduces the simple $\alpha=1 / 2$ case from before. The unique critical wind solution is then determined by the (appropriate) saddle point of the function

$n(s, \theta) \sim \cos \theta\left(\frac{F_{s}}{g_{s}^{1-\alpha}}\right)^{1 / \alpha}$.

The effective gravitational acceleration (gravity minus centrifugal force) above a Keplerian accretion disk along the $s$-streamline is given by

$g_{s}(s)=-G M_{\mathrm{WD}} R\left[\frac{s / R+\sin \theta}{\left(R^{2}+2 R s \sin \theta+s^{2}\right)^{3 / 2}}-\frac{\sin \theta}{(R+s \sin \theta)^{3}}\right]$.

Closed algebraic expressions for the radiative flux can only be given in highly simplified cases for the radial temperature run in the disk, for example, for an isothermal disk and for $T \sim r^{-1 / 2}$, see Feldmeier \& Shlosman (1999). The disk with a $T \sim r^{-1 / 2}$ temperature stratification is called Newtonian in the following. Fortunately, the radiation field from a narrow ring emitting as a black body can be calculated analytically, and thus the disk emission be obtained from a 1D numerical integration. The nozzle function obtained from this, along with the relevant saddle points, is shown in Fig. 6 of Feldmeier \& Shlosman (1999), and the relevant eigenvalues for $\theta$ are given in their Table 1 . Note that $\lambda_{\mathrm{cr}}=90^{\circ}-\theta_{\mathrm{c}}$ is tabulated there. The value of the nozzle function itself at the saddle, that is, the critical mass-loss rate, is given in the same table, and is shown as function of footpoint radius in Fig. 3. Note that in Shlosman \& Vitello (1993), the mass-loss rate from a disk ring is taken as proportional to the ring area $R \mathrm{~d} R$. This is a severe approximation, and the current eigenvalue or nozzle analysis allows us to derive the mass-loss rate from a ring as a function of its radius.

The wind starts roughly at three WD radii $\left(R_{\mathrm{WD}}\right)$ from the disk center. Farther in, details of wind launching are currently unknown because of the complexity of the disk boundary layer, the disk corona, etc. Interestingly, in the wind models of Feldmeier \& Shlosman (1999), who adopted a Shakura \& Sunyaev (1973) $\alpha$-disk, no appropriate saddle point of the nozzle function and hence no critical wind solution is found inside $\approx 3 R_{\mathrm{WD}}$.

For the outer wind termination radius in the disk, we do not follow the conclusion in Feldmeier et al. (1999) that the total mass-loss rate is dominated by the inner wind up to $7 R_{\mathrm{WD}}$, for instance, and that regions farther out can be neglected. Instead, Fig. 3 indicates that the region in the disk from ten to thirty

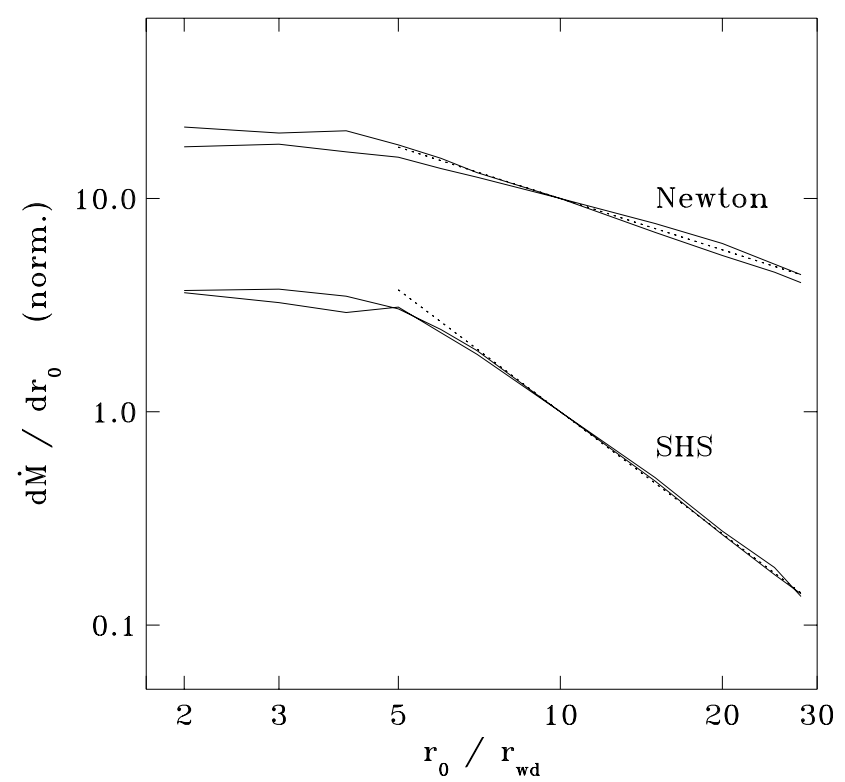

Fig. 3. Mass-loss rate per disk annulus, $\mathrm{d} \dot{M}_{\text {wind }} / \mathrm{d} r_{0}$, as a function of the footpoint radius. Data for $\alpha=2 / 3$ and $\alpha=1 / 2$ were used for both Newtonian and $\alpha$-disks. Fits with $\mathrm{d} \dot{M}_{\text {wind }} / \mathrm{d} r_{0} \propto r_{0}^{-1.9}(\alpha$-disk) and $\propto r_{0}^{-0.8}$ (Newtonian disk) are shown as dotted lines. Curves are nomalized at $r_{0} / r_{\mathrm{WD}}=10$. This is Fig. 2 from Feldmeier, A., Shlosman, I., \& Vitello, P. (1999).

$R_{\mathrm{WD}}$ should contribute roughly one third of the total mass-loss rate. For $r>5 R_{\mathrm{WD}}$, Feldmeier et al. (1999) found a power-law dependence

$\mathrm{d} \dot{M}_{\text {wind }} / \mathrm{d} R=R^{-\lambda}$.

An approximate value for $\lambda$ can be estimated from Eq. (19) by considering the $R$-dependence of the nozzle function in the near disk regime $(s \rightarrow 0)$. Obviously then, gravity $g \sim R^{-2}$, and flux $F$ resp. intensity $I F \sim I^{4} \sim R^{-2}$ to $R^{-3}$, where in the latter relation the outer regions of either a Newtonian or $\alpha$-disk are assumed. From Eq. (19), $\mathrm{d} \dot{M}_{\text {wind }} / \mathrm{d} R \sim R^{-1}$ follows (in Feldmeier et al. 1999: $R^{-0.8}$ ) for the Newtonian disk and either $\alpha=1 / 2$ or $2 / 3$; and $\mathrm{d} \dot{M}_{\text {wind }} / \mathrm{d} R \sim R^{-3}$ resp. $R^{-2.5}$ for the $\alpha$-disk and $\alpha=1 / 2$ resp. $2 / 3$ (in Feldmeier et al. 1999: $R^{-1.9}$ for both $\alpha$ ). In our wind models, we adopted the values for $\lambda$ from Feldmeier et al. (1999).

The critical angle $\theta_{c}$ varies only marginally in the windlaunching area. We adopted the values for the different disk models calculated by Feldmeier \& Shlosman (1999) as shown in their Table 1 . For $r_{0} \approx 3 R_{\mathrm{WD}}$ they found $\theta_{\mathrm{c}}=25^{\circ} \ldots 27^{\circ}$ and for the outer edge of the wind at $r_{0} \approx 30 R_{\mathrm{WD}}$ they found $\theta_{\mathrm{c}}=33^{\circ} \ldots 39^{\circ}$. The influence on the resulting spectra of such a minor variation in $\theta_{\mathrm{c}}$ is negligible compared with the other uncertainties in the model. We therefore adopted a simple linear variation of $\theta_{\mathrm{c}}$ from $25^{\circ}$ to $35^{\circ}$ as $r_{0}$ varies from $3 R_{\mathrm{WD}}$ to $30 R_{\mathrm{WD}}$.

\section{Monte Carlo radiative transfer}

We have developed a Monte Carlo based code to calculate radiative transfer in three dimensions (WoMPAT). The two wind models described above are implemented. We use photon packets with energy weights in our implementation, where $w_{i}$ represents the weight of the $i$-th photon. A weight of $w=1$ corresponds to an energy

$\Delta E=\frac{E_{\mathrm{tot}}}{N_{\mathrm{pp}}}$ 
where $N_{\mathrm{pp}}$ corresponds to the total number of photons and $E_{\mathrm{tot}}$ to the total energy of all photons used in the simulation. We also use the weights as a method of keeping the Monte Carlo noise equal for each frequency bin, therefore the weights have to be adjusted as

$w_{v}=\frac{E_{v}}{n_{\mathrm{pp}}} \frac{1}{\Delta E}$

where $E_{v}$ is the total energy in the frequency bin $v$ and $n_{\mathrm{pp}}$ the number of photons in that bin, the factor $1 / \Delta E$ normalizes the weight with respect to the $w=1$ case. Because we then use exactly the same number of photons for each frequency bin, the initial frequency bin is not randomly sampled, only the initial photon frequency in this bin is sampled from a linear distribution.

Photons are either created on the WD with a probability $p_{\mathrm{WD}}=E_{\mathrm{WD}} / E_{\mathrm{tot}}$ or at the disk surface with a probability $p_{\text {disk }}=E_{\text {disk }} / E_{\text {tot }}$. A boundary layer as radiation source is not included. If a photon is created on the WD, the initial position on the WD surface is completely random. The initial direction is determined with respect to the local outward normal and then transformed back to the standard coordinate system with the origin at the WD center. In local polar coordinates, the initial direction is given by $\hat{\boldsymbol{d}}_{\text {init }}=(1, \theta, \phi)$. The probability to emit in a solid angle $\mathrm{d} \omega=\sin \theta \mathrm{d} \theta \mathrm{d} \phi$ is given by

$\mathrm{d} p(\theta, \phi)=\eta(\theta) \cos \theta \sin \theta \mathrm{d} \phi \mathrm{d} \theta$,

where $\eta(\theta)$ is the standard limb darkening in Eddington approximation and $\cos \theta$ is a geometric factor accounting for the foreshortening of the emitting surface. The $\sin \theta$ factor is purely due to the spherical coordinates and later again comes into play when the flux per solid angle $I_{v} / \mathrm{d} \omega$ is calculated from the photon bins. Obviously, the $\phi$-direction is arbitrary and as such is sampled by $\phi=\zeta 2 \pi$, where $\zeta$ is a pseudo-random number drawn from a uniform distribution between zero and one.

If a photon is created on the disk surface, the initial position is given by $\boldsymbol{r}_{\text {init }}=\left(r_{\mathrm{i}}, \phi_{\mathrm{i}}, z_{\mathrm{i}}\right)$ in cylindrical coordinates. It is $z_{\mathrm{i}}=0$ because the disk is taken to be infinitesimally thin, furthermore, the disk is axisymmetric, thus $\phi_{\mathrm{i}}=\zeta 2 \pi$. The initial radial position $r_{\mathrm{i}}$ is found via a lookup table from the cumulative distribution function

$P\left(r_{\mathrm{i}}\right)=\frac{\int_{r_{\text {inner }}}^{r_{\mathrm{i}}} I_{v} 2 \pi r \mathrm{~d} r}{\int_{r_{\text {inner }}}^{r_{\text {disk }}} I_{v} 2 \pi r \mathrm{~d} r}$,

where $r_{\text {disk }}$ is the radius of the accretion disk and $I_{v}=I_{v}(\theta=0)$ the intensity in the frequency bin at $v$. The initial direction $\hat{d}$ is found in the same way as for emission on the WD.

The next step is to determine whether and where a photon interacts. For this the optical depth along the line of flight (LOF) is calculated by integrating the opacities via a trapezium rule with transformations into the co-moving frame at each integration point. Velocity gradients are taken into consideration. Opacities include bound-bound, bound-free, free-free, and electron scattering, although it was found that only the line opacities are important. All opacities are computed in LTE approximation. Generalization to NLTE is devoted to future work.

After this integration we not only know the total optical depth $\tau_{\text {tot }}$ for this LOF in the medium, but also the run of the optical depth $\tau=\tau(s)$ as a function of the distance $s$ along the LOF. From the definition of the optical depth, the probability that the photon escapes from the medium is given by

$p_{\text {esc }}=\mathrm{e}^{-\tau_{\text {tot }}}$, then the probability that the photon scatters is given by

$p_{\text {scat }}=1-p_{\text {esc }}$.

If the sampled random number $\zeta<p_{\text {esc }}$, then the photon escapes the medium, otherwise it scatters at an optical depth of

$\tau_{\text {scat }}=-\ln \left[1-\zeta\left(1-\mathrm{e}^{-\tau_{\text {tot }}}\right)\right]$.

After calculating $\tau_{\text {scat }}$, the point $s$ along the LOF (and thus the location $\boldsymbol{r}_{\text {scat }}$ ) where this optical depth is reached and the scattering process occurs is easily determined by inverting $\tau(s)$.

The escape of the photon can be implemented in two ways. The straightforward way is to really determine whether the photon leaves the wind or not, and if it leaves the wind, then it really leaves the simulation volume and "dies", if it remains in the wind, the whole photon weight remains in the wind. This is called a "live or die" algorithm. On the other hand, the notion of weights allows at this point for an algorithm referred to as "forced scattering". A part of the photon is taken to always scatter in the medium and a part of the photon, namely the one with the weight $w_{\text {esc }}=w p_{\text {esc }}$, escapes and is detected. In this case $\zeta$ does not determine if scattering occurs, but only determines the point where scattering for the photon with the reduced weight $w_{\text {new }}=w p_{\text {scat }}$ occurs via Eq. (29). This technique is based on the assumption that a photon is really a photon packet consisting of many photons out of which a certain part would actually exhibit this behavior and escape and another part would remain in the medium. Such an algorithm is used to reduce the noise of the simulation and has been used in a Monte Carlo simulation of accretion disk winds by Knigge et al. (1995).

After the scattering location is known, and also whether and in which line the scattering occurs, which is of course not determined probabilistically, all there remains to be done is to assign the photon a new frequency $v_{\text {new }}$ and a new direction $\hat{\boldsymbol{d}}_{\text {new }}$, both are taken to be independent of the incident $v_{\text {old }}$ and $\hat{\boldsymbol{d}}_{\text {old }}$. For line scattering the new direction is drawn from a spherically isotropic distribution and the new frequency is drawn from a Gaussian in the co-moving frame and then transformed into the rest frame. Therefore the new frequency is given by

$v_{\text {new }}=\left(v_{0}+\xi \Delta v_{\mathrm{D}}\right)\left[1+\frac{\boldsymbol{v}\left(\boldsymbol{r}_{\text {scat }}\right) \cdot \hat{\boldsymbol{d}}_{\text {new }}}{c}\right]$,

where $\xi$ is a random number sampled from a Gaussian distribution with mean zero and standard deviation unity, $c$ is the speed of light, and $v_{0}$ and $\Delta v_{\mathrm{D}}$ are the rest frequency and the Doppler width of the line in which scattering occurs, respectively. Our main concerns are strong resonance lines, therefore we take the line scattering as $100 \%$ efficient. Bound-free and free-free processes are ignored, that is, only electron scattering is taken into account. It is assumed to be coherent and isotropic.

Using a Sobolev approach would be computationally less expensive, but there are some limitations. Multiple scattering in the resonance region is neglected, there is only one interaction per resonance surface. In wind situations with a non-monotonic velocity law Sobolev calculations are still feasible, but become more complicated. As the resonance surfaces are taken to be sharp, physical quantities are assumed to be constant on the resonance surface. The assumption of a finite resonance region in which physical quantities are allowed to change is more realistic. In cases where more effects such as line overlap or wind clumping have to be considered, it is better to drop the Sobolev approximation and adopt accurate methods such as co-moving frame calculations (see e.g. Hillier et al. 2003). This method is based on a transformation into the co-moving frame at which the 
local medium is at rest. Opacities and emissivities are isotropic in this frame, the anisotropy is taken care of by the frame transformation. Numerical integration of the radiative transfer equation, or integration of the opacities, requires a frame transformation at every sampling point with this method (see e.g. Knigge et al. 1995). Such a method is more expensive, but can reveal differences with respect to the Sobolev method, for example, a comparison of co-moving frame methods and Sobolev methods for $\mathrm{O}$ and WR star winds yield differences in wind acceleration and clumping factors (Hillier et al. 2003; Gräfener \& Hamann 2005; and Gräfener 2007, priv. comm.). In a Monte Carlo context opacity calculations using a co-moving frame method are a tractable problem (Knigge et al. 1995), where the trade-off in run time with current computer power to the gained insight from a comparison with older Sobolev results is obvious. We therefore implemented a co-moving frame method in this work.

\section{Accretion disk code AcDc}

To calculate the metal-line blanketed NLTE accretion-disk models, which are input models for the wind calculations, we used our accretion-disk code AcDc (Nagel et al. 2004). It is based on the radial structure of an $\alpha$-disk (Shakura \& Sunyaev 1973), assuming a stationary, geometrically thin disk (the total disk thickness $H$ is much smaller than the disk diameter). This allows the decoupling of the vertical and radial structures and, assuming axial symmetry, we can separate the disk into concentric annuli of plane-parallel geometry. In that way, the radiative transfer becomes a $1 \mathrm{~d}$ problem. The radial distribution of the effective temperature $T_{\text {eff }}$ can be described by

$T_{\mathrm{eff}}(R)=\left[\frac{3 G M_{\mathrm{WD}} \dot{M}_{\text {disk }}}{8 \pi \sigma R^{3}}\left(1-\sqrt{\frac{R_{\mathrm{WD}}}{R}}\right)\right]^{1 / 4}$,

where $\dot{M}_{\text {disk }}$ denotes the mass-accretion rate through the disk and $\sigma$ is the Stefan-Boltzmann constant.

For each disk ring, the following set of coupled equations were solved simultaneously under the constraints of particle number and charge conservation:

- radiation transfer for the specific intensity $I$,

- hydrostatic equilibrium between gravitation, gas pressure $P_{\text {gas }}$, and radiation pressure,

- energy balance between the viscously generated energy $E_{\text {mech }}$ and the radiative energy loss $E_{\text {rad }}$,

- NLTE rate equations for the population numbers $n_{i}$ of the atomic levels $i$.

By integrating the spectra of the individual annuli, one obtains a complete disk spectrum for different inclination angles, where the spectral lines are Doppler shifted according to the radial component of the Kepler rotational velocity. For a more detailed description of the accretion-disk modeling with AcDc, see Nagel et al. (2004).

\section{Spectral properties of kinematical and hydrodynamic models}

We tested the influence of the wind temperatures as well as the influence of the size of the computational domain on the calculated spectrum in the case of a standard SV (Table 1) and a standard hydrodynamic model (Table 2).

\subsection{Standard SV model}

The calculated spectra for a constant wind temperature of $20000 \mathrm{~K}$ show a very prominent $\mathrm{C}_{\mathrm{IV}}$ line at $1550 \AA$ A. Depending on the inclination angle, it shows an absorption, emission, or
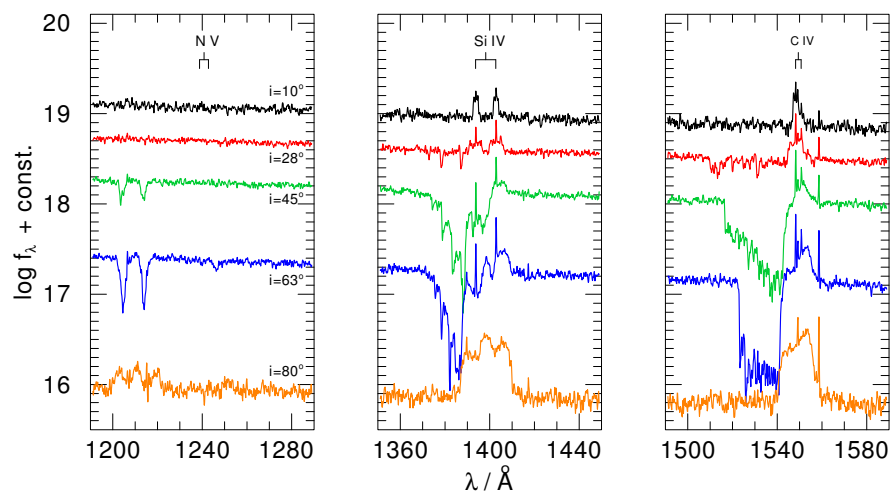

Fig. 4. Standard SV model spectra for $T_{\text {wind }}=20000 \mathrm{~K}$ on a large domain. Note the prominent C IV $1550 \AA$ and Si Iv $1400 \AA$ lines while Nv $1240 \AA$ is absent.

Table 1. Standard parameter set for the SV model.

\begin{tabular}{lclc}
\hline \hline Parameter & Value & Parameter & Value \\
\hline$M_{\mathrm{WD}}\left(M_{\odot}\right)$ & 0.8 & $R_{\mathrm{WD}}(\mathrm{cm})$ & $7 \times 10^{8}$ \\
$T_{\mathrm{WD}}(\mathrm{K})$ & 40000 & $\dot{M}_{\text {disk }}\left(M_{\odot} \mathrm{yr}^{-1}\right)$ & $1 \times 10^{-8}$ \\
$R_{\text {disk }}\left(R_{\mathrm{WD}}\right)$ & 34 & $\dot{M}_{\text {wind }}\left(M_{\odot} \mathrm{yr}^{-1}\right)$ & $1 \times 10^{-9}$ \\
$\theta_{\min }(\mathrm{deg})$ & 20 & $\theta_{\max }(\mathrm{deg})$ & 65 \\
$r_{\min }\left(R_{\mathrm{WD}}\right)$ & 4 & $r_{\max }\left(R_{\mathrm{WD}}\right)$ & 12 \\
$R_{v}\left(R_{\mathrm{WD}}\right)$ & 100 & $v_{\infty}\left(v_{\mathrm{esc}}\right)$ & 3 \\
$\alpha$ & 1.5 & $\lambda$ & 0 \\
\hline
\end{tabular}

Table 2. Standard parameter set for the hydrodynamic wind model.

\begin{tabular}{lclc}
\hline \hline Parameter & Value & Parameter & Value \\
\hline$M_{\mathrm{WD}}\left(M_{\odot}\right)$ & 0.8 & $R_{\mathrm{WD}}(\mathrm{cm})$ & $7 \times 10^{8}$ \\
$T_{\mathrm{WD}}(\mathrm{K})$ & 40000 & $\dot{M}_{\text {disk }}\left(M_{\odot} \mathrm{yr}^{-1}\right)$ & $1 \times 10^{-8}$ \\
$R_{\mathrm{disk}}\left(R_{\mathrm{WD}}\right)$ & 34 & $\dot{M}_{\text {wind }}\left(M_{\odot} \mathrm{yr}^{-1}\right)$ & $1 \times 10^{-9}$ \\
$\theta_{\min }(\mathrm{deg})$ & 25 & $\theta_{\max }(\mathrm{deg})$ & 35 \\
$r_{\min }\left(R_{\mathrm{WD}}\right)$ & 3 & $r_{\max }\left(R_{\mathrm{WD}}\right)$ & 30 \\
$R_{v}\left(R_{\mathrm{WD}}\right)$ & 100 & $v_{\infty}\left(v_{\mathrm{esc}}\right)$ & 3 \\
$\alpha$ & 1.5 & $\lambda$ & 0 \\
\hline
\end{tabular}

Notes. $r_{\min }, r_{\max }, \theta_{\min }$, and $\theta_{\max }$ are no longer free parameters, but fixed by the nozzle analysis.

P Cygni profile. Another strong feature is the Si Iv line at $1400 \AA$, showing the same profiles as the carbon line. The $\mathrm{Nv}$ line at $1240 \AA$ is missing because of the low wind temperature (Fig. 4 , all calculated spectra here and in the following figures of this section are folded with a $0.3 \AA$ Gaussian.).

For a constant wind temperature of $40000 \mathrm{~K}$ the strength of the $\mathrm{C}$ IV and Si IV lines decrease, but the Nv line at $1240 \AA$ is strong and shows a P Cygni profile for intermediate inclination angles (Fig. 5).

Different sizes of the computational domain affect the spectra (Fig. 6, lower panel). The width of the absorption troughs is broader for the larger domain (cylinder with radius and height of $2000 R_{\mathrm{WD}}$ ) than for the small domain (cylinder with radius and height of $100 R_{\mathrm{WD}}$ ). We conclude that there is absorbing material in the lines even beyond the small domain. These faster-moving parts of the wind are missed using the small domain, and hence, the lines are narrower. Therefore, it is necessary to use the larger domain. For an even larger domain of $20000 R_{\mathrm{WD}}$, the width of the absorption troughs does not change any further than for the $2000 R_{\text {WD }}$ case. A grid with $100 \times 100$ boxes resolves the lines slightly better than a $30 \times 30$ grid (Fig. 6, upper panel), but the significantly higher demands in memory and computation time are not justified by the small difference. 
D.-J. Kusterer et al.: Monte Carlo radiation transfer in CV disk winds
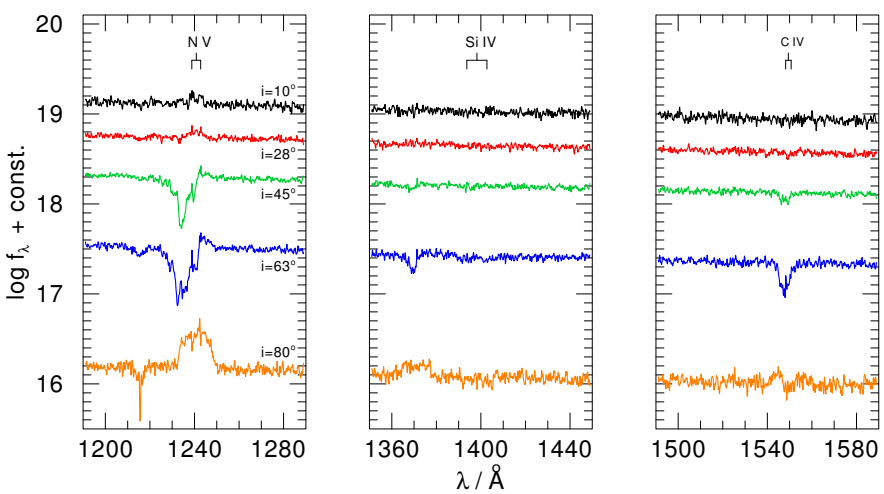

Fig. 5. Same as Fig. 4, but for $T_{\text {wind }}=40000 \mathrm{~K}$. Note the prominent N v $1240 \AA$ A line, while C Iv $1550 \AA$ and Si Iv $1400 \AA$ are very weak.

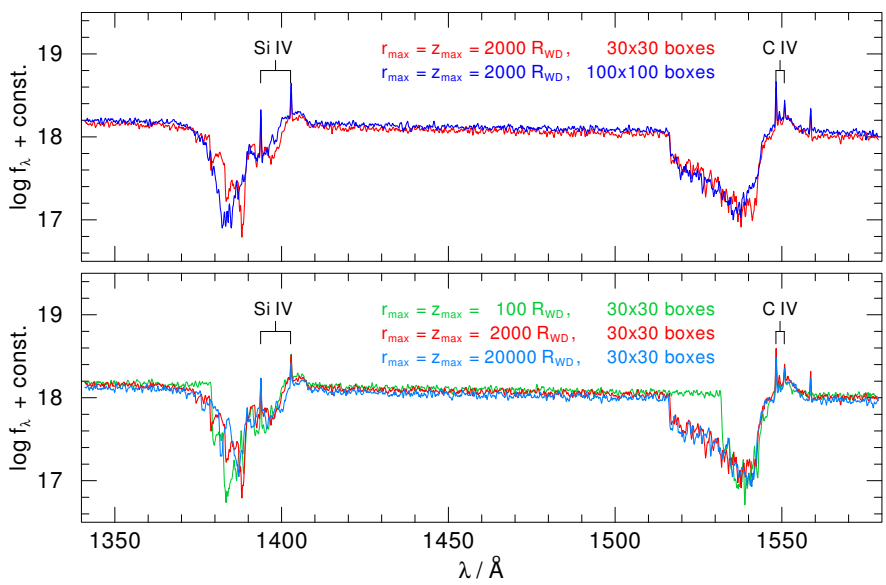

Fig. 6. Comparison of the CIV $1550 \AA$ and Si IV $1400 \AA$ lines for SV models with $20000 \mathrm{~K}$ wind temperature, different computational domain sizes (lower panel), and different numbers of boxes (upper panel) for $i=45^{\circ}$. Note, that the lines for the larger domain are broader.

Especially for the cooler model, the spectra show narrow emission and absorption spikes. They are limited to only one frequency point at the rest wavelength of a spectral line. We interpret them as numerical artifacts, but their origin remains unclear.

\subsection{Standard hydrodynamic model}

The calculations for the standard hydrodynamic model show similar results as for the standard SV model. The main difference for a constant wind temperature of $20000 \mathrm{~K}$ is that the C IV $1550 \AA$ and Si IV $1400 \AA$ lines have a narrower absorption trough and show a double-peaked profile for high inclination angles (Fig. 7). For a constant wind temperature of $40000 \mathrm{~K}$ the N v $1240 \AA$ line is narrower than in the SV model (Fig. 8). These differences are probably due to the higher collimation of the wind for the hydrodynamic model. Artificial spikes as in the $\mathrm{SV}$ model are much less pronounced.

\section{AM CVn: wind modeling}

AMCVn, the prototype system for the class of AMCVn stars, is a hydrogen-deficient nova-like CV (Downes et al. 1997). The orbital period is $1029 \mathrm{~s}$ (Harvey et al. 1998). For primary and secondary masses there are several values in discussion, with the most agreed upon being $M_{\mathrm{WD}} \approx 0.7 M_{\odot}$ and $M_{2} \approx 0.1 M_{\odot}$ (Roelofs et al. 2007). However, using synthetic spectra of a large grid of accretion-disk models (Nagel et al. 2009) calculated with AcDc, we found the best agreement with observation for models with $M_{\mathrm{WD}}=0.6 M_{\odot}$ and an accretion rate $\dot{M}=1 \times 10^{-8} M_{\odot} \mathrm{yr}^{-1}$. Therefore these parameters were adopted
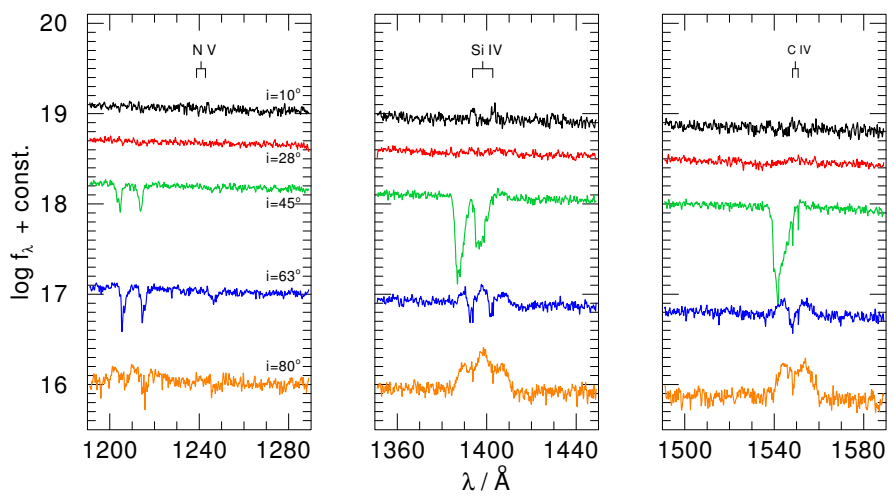

Fig. 7. Same as Fig. 4, but for the standard hydrodynamic model.
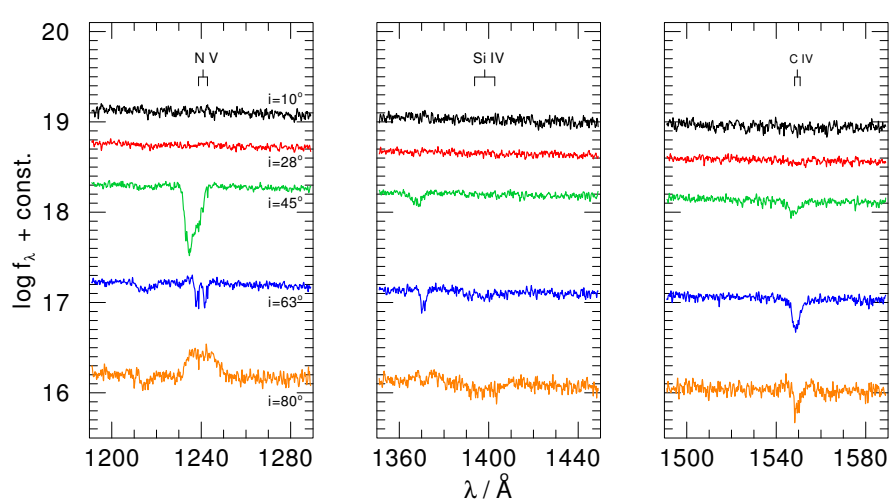

Fig. 8. Same as Fig. 5, but for the standard hydrodynamic model.

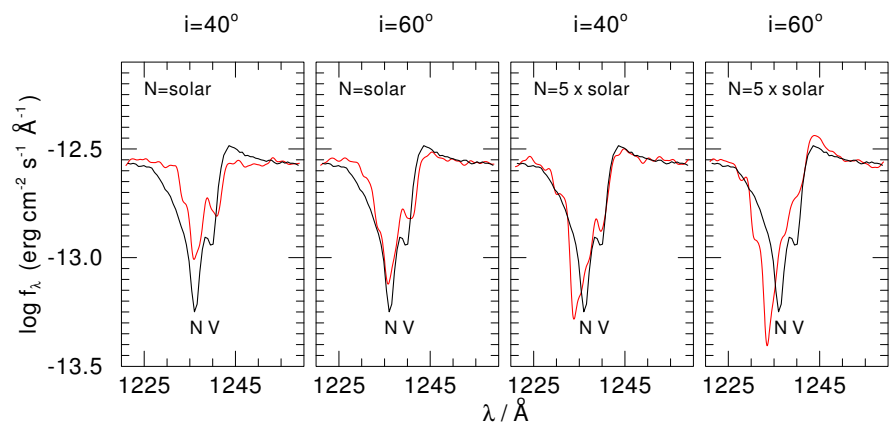

Fig. 9. Comparison of the calculated $\mathrm{N} v$ resonance line (SV model) with an observed spectrum of AM CVn. The STIS observation is plotted in black in each panel, the calculated lines $\left(T_{\text {wind }}=40000 \mathrm{~K}, \dot{M}_{\text {wind }}=\right.$ $\left.1 \times 10^{-9} M_{\odot} \mathrm{yr}^{-1}\right)$ are drawn in red.

for the study of AMCVn wind spectra in this work. The corresponding WD radius is $R_{\mathrm{WD}}=9550 \mathrm{~km}$. As accretion-disk extension we considered $r_{\text {in }}=1.36 R_{\mathrm{WD}}$ to $r_{\mathrm{out}}=14.66 R_{\mathrm{WD}}$, where the outer radius $r_{\text {out }}$ of the disk corresponds roughly to the tidal radius. The best agreement with observations for the disk models is found for an inclination of about $40^{\circ}$ (Nagel et al. 2004; Roelofs et al. 2007).

We used an SV model for the wind calculations with the parameter set as given in Table 3 . The chemical composition is helium-dominated with solar abundances of $\mathrm{C}, \mathrm{O}$, and $\mathrm{Si}$, two different values for $\mathrm{N}$, and no hydrogen. The calculations were performed on the high-performance cluster of the University of Tübingen, using up to 64 cores and 200 million photon packages. We compared our calculated model spectra with an observed spectrum taken by HST/STIS (Wade et al. 2007).

A first model with a constant wind temperature of $40000 \mathrm{~K}$ and either solar (left two panels) or five times solar $\mathrm{N}$ abundance (right two panels) is shown in Fig. 9. The wind model spectra indicate the observed P Cygni profile of the $\mathrm{N} v$ doublet around 


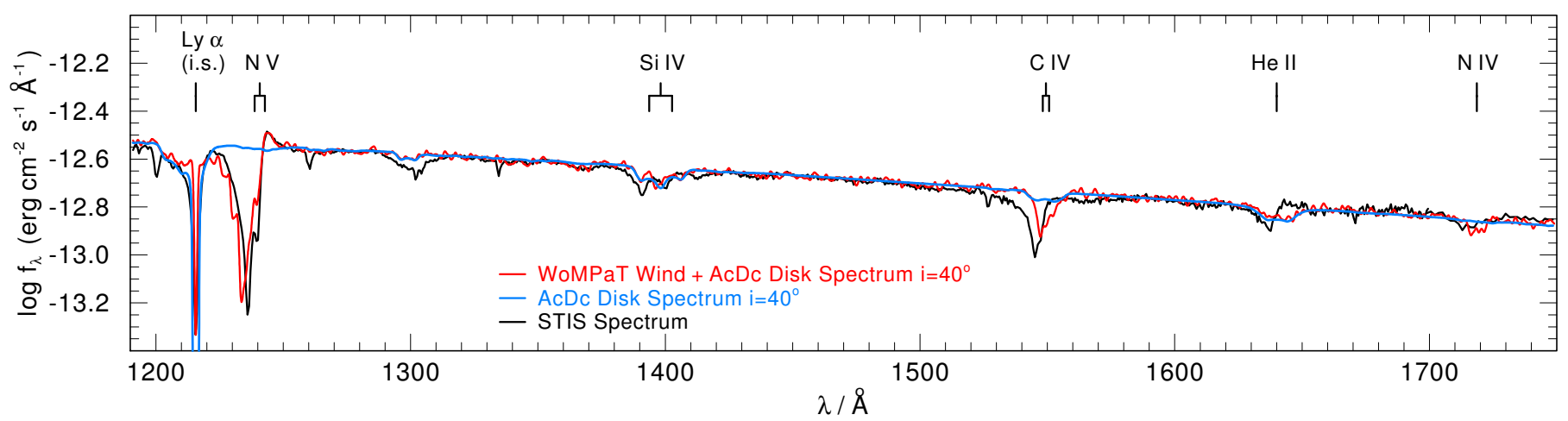

Fig. 10. HST/STIS spectrum of AM CVn (black), disk spectrum (blue), and wind spectrum (red, $T_{\text {wind }}=35000 \mathrm{~K}, \dot{M}_{\text {wind }}=5 \times 10^{-10} M_{\odot} \mathrm{yr}^{-1}$, SV model). Note the prominent P Cygni profile of the Nv $1240 \AA$ line is not similar to that of the disk, but to that of the wind model. All models are reddened by $E(B-V)=0.12$, and folded with a $1.2 \AA$ Gaussian.

Table 3. Parameter set of the SV wind model for AMCVn.

\begin{tabular}{lc}
\hline \hline Parameter & Value \\
\hline$M_{\mathrm{WD}}\left(M_{\odot}\right)$ & 0.6 \\
$R_{\mathrm{WD}}(\mathrm{cm})$ & $9.55 \times 10^{8}$ \\
$T_{\mathrm{WD}}(\mathrm{K})$ & 100000 \\
$\dot{M}_{\text {disk }}\left(M_{\odot} \mathrm{yr}^{-1}\right)$ & $1 \times 10^{-8}$ \\
$r_{\text {in }}\left(R_{\mathrm{WD}}\right)$ & 1.36 \\
$r_{\text {out }}\left(R_{\mathrm{WD}}\right)$ & 14.66 \\
$\dot{M}_{\text {wind }}\left(M_{\odot} \mathrm{yr}^{-1}\right)$ & $1 \times 10^{-9}, 5 \times 10^{-9}, 5 \times 10^{-10}$ \\
$\theta_{\min }(\mathrm{deg})$ & 20 \\
$\theta_{\max }(\mathrm{deg})$ & 65 \\
$r_{\min }\left(R_{\mathrm{WD}}\right)$ & 1.4 \\
$r_{\max }\left(R_{\mathrm{WD}}\right)$ & 10 \\
$R_{v}\left(R_{\mathrm{WD}}\right)$ & 100 \\
$v_{\infty}\left(v_{\mathrm{esc}}\right)$ & 3 \\
$\alpha$ & 1.5 \\
$\lambda$ & 0 \\
\hline
\end{tabular}

$1240 \AA$, but neither the model for an inclination of $i=40^{\circ}$, nor the model for $i=60^{\circ}$ gives a satisfying fit for a mass-loss rate of $1 \times 10^{-9} M_{\odot} \mathrm{yr}^{-1}$. In both cases the computed profile is not broad and deep enough. We chose these two inclinations because the first is the most probable value discussed in the literature and the latter is the most extreme inclination that might still be possible. As for some AMCVn systems a high abundance of nitrogen is reported, for example, GP Com (Morales-Rueda et al. 2003) or V396 Hya (Ramsay et al. 2006), we calculated a wind spectrum with the nitrogen abundance increased to five times solar. The resulting $\mathrm{N} v$ line is generally deeper, the model matches the observed spectrum better for $i=40^{\circ}$. However, one has to keep in mind that there are other parameters that might influence the resulting spectrum, for instance, those describing the wind geometry, which have not been investigated, as well as other nitrogen abundances.

A closer comparison of the Nv $1240 \AA$ doublet and the C IV $1550 \AA$ doublet with the observed STIS spectrum was performed for the $30 \times 30$ grid on the large computational domain for four different wind temperatures $(30000 \mathrm{~K}, 35000 \mathrm{~K}$, $40000 \mathrm{~K}$, and $45000 \mathrm{~K})$ and for three mass-loss rates $\left(5 \times 10^{-10}\right.$, $1 \times 10^{-9}$, and $\left.5 \times 10^{-9} M_{\odot} \mathrm{yr}^{-1}\right)$. The nitrogen abundance was set to five times solar. Results for $i=40^{\circ}$ are plotted in Figs. 12 and 13, those for $i=60^{\circ}$ in Figs. 14 and 15. For $i=40^{\circ}$, the $\mathrm{Nv}$ line is best reproduced by combining a wind temperature of $40000 \mathrm{~K}$ and a mass-loss rate of $1 \times 10^{-9} M_{\odot} \mathrm{yr}^{-1}$ or a wind temperature of $35000 \mathrm{~K}$ and a mass-loss rate of $5 \times 10^{-10} M_{\odot} \mathrm{yr}^{-1}$.
For higher and lower wind temperatures, the line is too weak. Furthermore, a higher mass-loss rate results in a too broad absorption trough. For $i=60^{\circ}$, the same is true: the best agreement with the observation is found for a wind temperature of $40000 \mathrm{~K}$ and a mass-loss rate of $5 \times 10^{-10} M_{\odot} \mathrm{yr}^{-1}$. Generally, the spectral line is broader and deeper than for $i=40^{\circ}$.

For the C IV line, the lowest wind temperature can be excluded for all mass-loss rates and both inclinations because the resulting line profile is too deep. Moreover, none of the models for $i=60^{\circ}$ and wind temperature of $35000 \mathrm{~K}$ or highest mass-loss rate results in satisfying line profiles. For $i=40^{\circ}$, a good agreement with observation is obtained for a wind temperature of $35000 \mathrm{~K}$ and a mass-loss rate between $5 \times 10^{-10}$ and $1 \times 10^{-9} M_{\odot} \mathrm{yr}^{-1}$.

The discrepancies in the parameter values needed to match the $\mathrm{C}_{\mathrm{IV}}$ and $\mathrm{Nv}$ lines indicate a non-isothermal wind structure, as shown for example by Long \& Knigge (2002) or Noebauer et al. (2010) for several CVs. A different mass-loss rate for different species is unlikely, the correct value is probably between $1 \times 10^{-9} M_{\odot} \mathrm{yr}^{-1}$ and $5 \times 10^{-10} M_{\odot} \mathrm{yr}^{-1}$.

In Fig. 10 we show the observed UV spectrum, our bestfit SV model for the wind $\left(\dot{M}_{\text {wind }}=5 \times 10^{-10} M_{\odot} \mathrm{yr}^{-1}\right.$, $\left.T_{\text {wind }}=35000 \mathrm{~K}, i=40^{\circ}\right)$, and an accretion-disk model. The wind model reproduces the observed spectrum much better than the pure disk spectrum. Both synthetic spectra are reddened by $E(B-V)=0.12$. Calculating a hydrodynamic model for the same parameters, the result looks similar. The C IV $1550 \AA$ line is deeper, matching the depth of the observed line better. Unfortunately, the model spectrum does not show the emission peak of the N v $1240 \AA$ line, which is well reproduced in the SV model.

Not all lines in the observed spectrum are produced by the accretion-disk wind. Figure 11 shows that with a multi color blackbody disk as input for the wind calculation especially the Si Iv line cannot be reproduced as well as with an NLTE accretion-disk spectrum as input, whereas the $\mathrm{Nv}$ line is a pure wind line.

\section{Conclusions}

We have performed 2.5D Monte Carlo radiative transfer calculations with LTE opacities in hydrodynamic wind structures using NLTE spectra from the accretion disk as input and accounting for the white dwarf as an additional photon source. Our main conclusions are listed below.

- Sampling the accretion-disk wind by a $30 \times 30$ grid is sufficient for a good resolution of the wind lines. But it 
D.-J. Kusterer et al.: Monte Carlo radiation transfer in CV disk winds

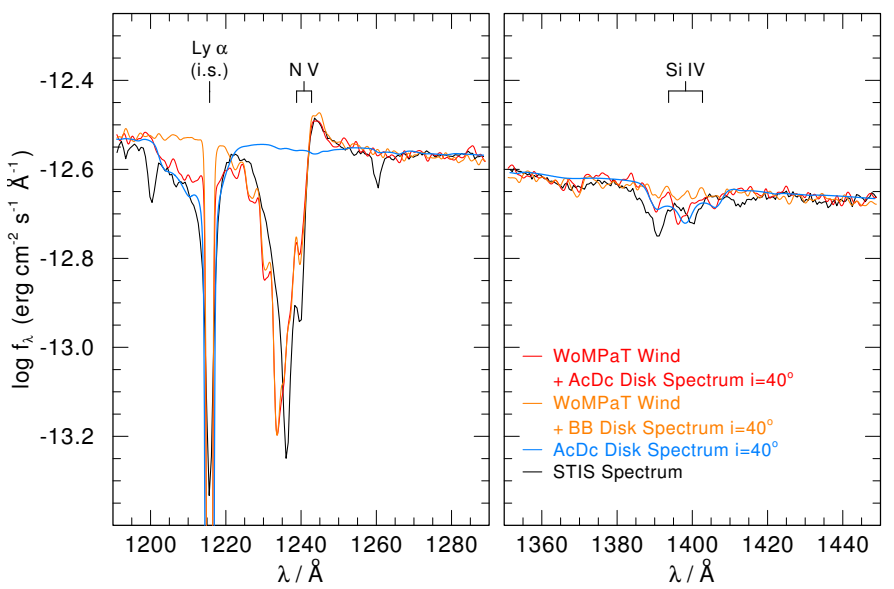

Fig. 11. HST/STIS spectrum of AM CVn (black) compared with a wind spectrum with an NLTE accretion-disk model spectrum as input (red) and a wind spectrum with a multi-color blackbody spectrum as input (orange). In blue, the pure NLTE disk spectrum is shown.

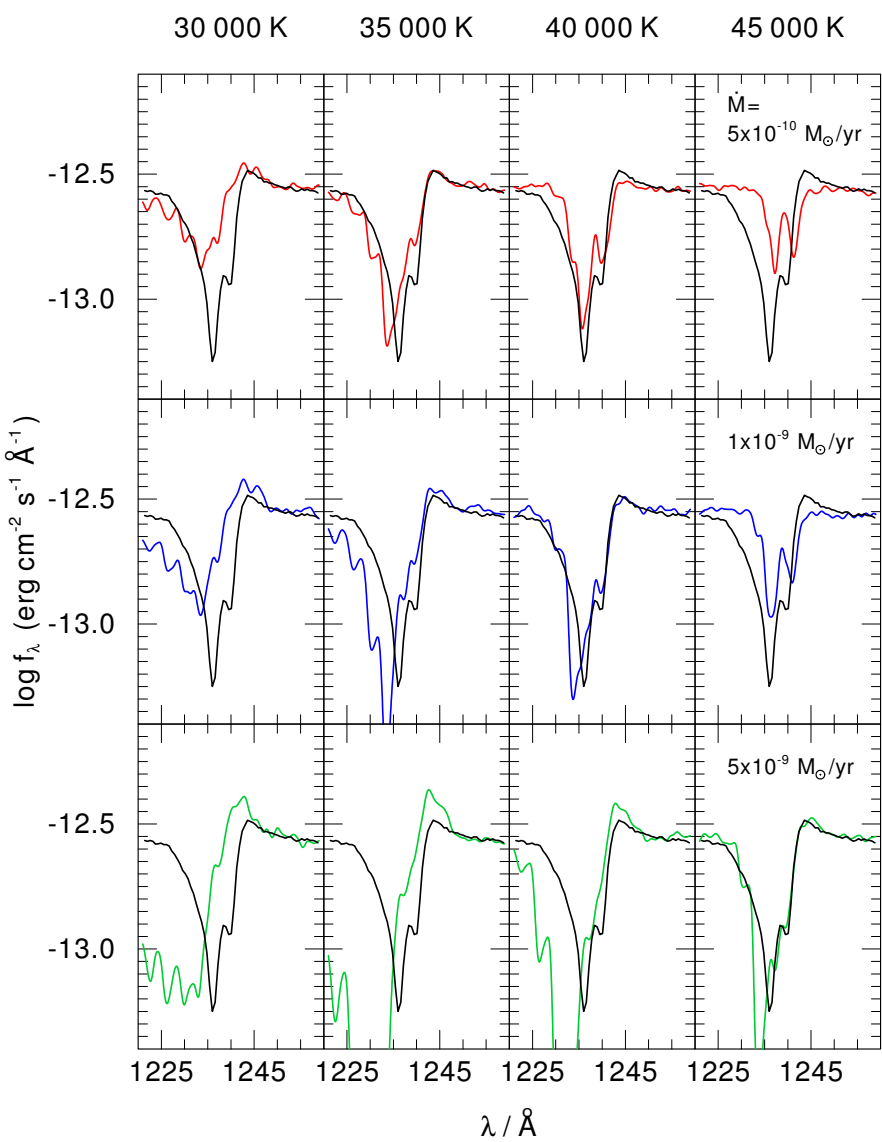

Fig. 12. Comparison of the calculated $\mathrm{Nv} 1240 \AA$ line $\left(i=40^{\circ}\right.$, SV model) with the STIS observation (black).

is necessary to extend the computational domain up to $2000 R_{\mathrm{WD}}$ instead of only a few hundred to include fastermoving absorbing material farther out that contributes significantly to the line widths.

- The two accretion-disk wind descriptions implemented in WoMPAT produce similar line profiles. The major difference between kinematical and hydrodynamic wind models are narrower lines in the hydrodynamic models due to the used wind geometry.

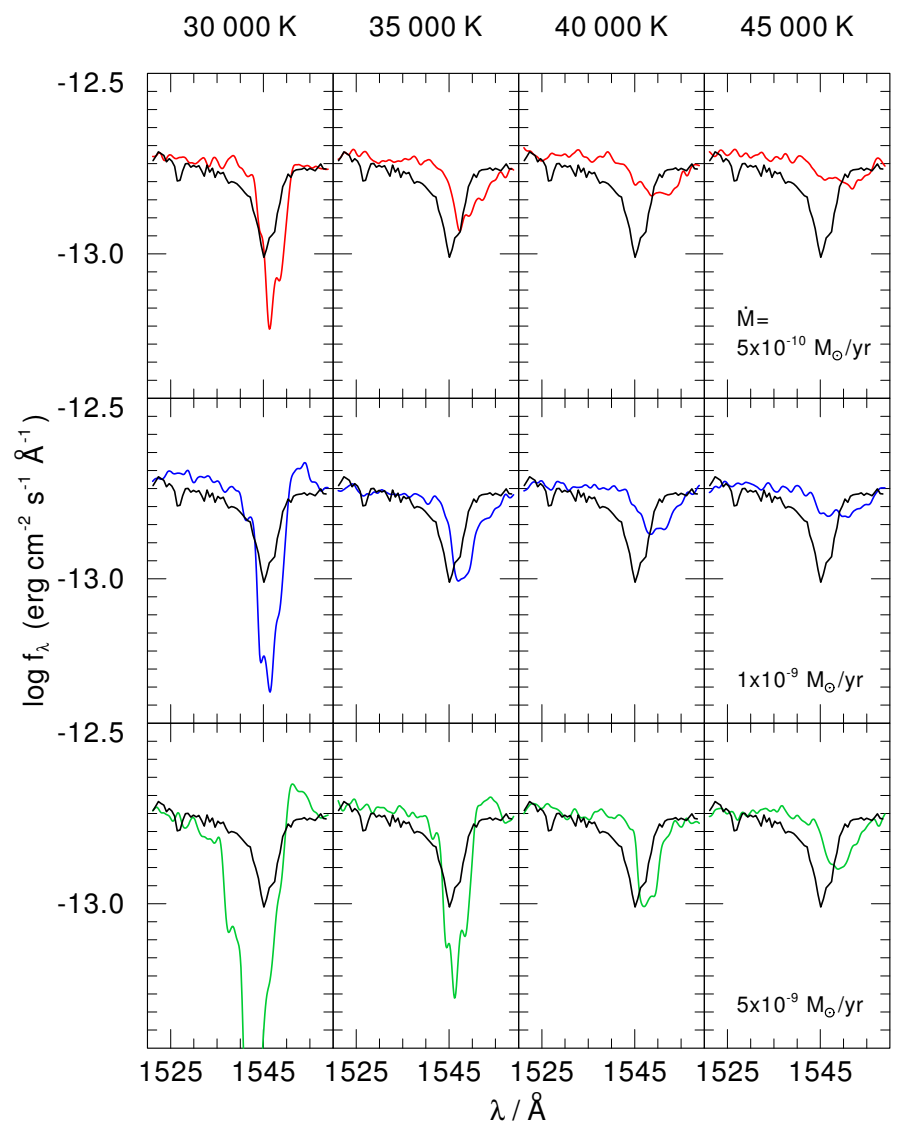

Fig. 13. Same as Fig. 12, but for C Iv $1550 \AA$.

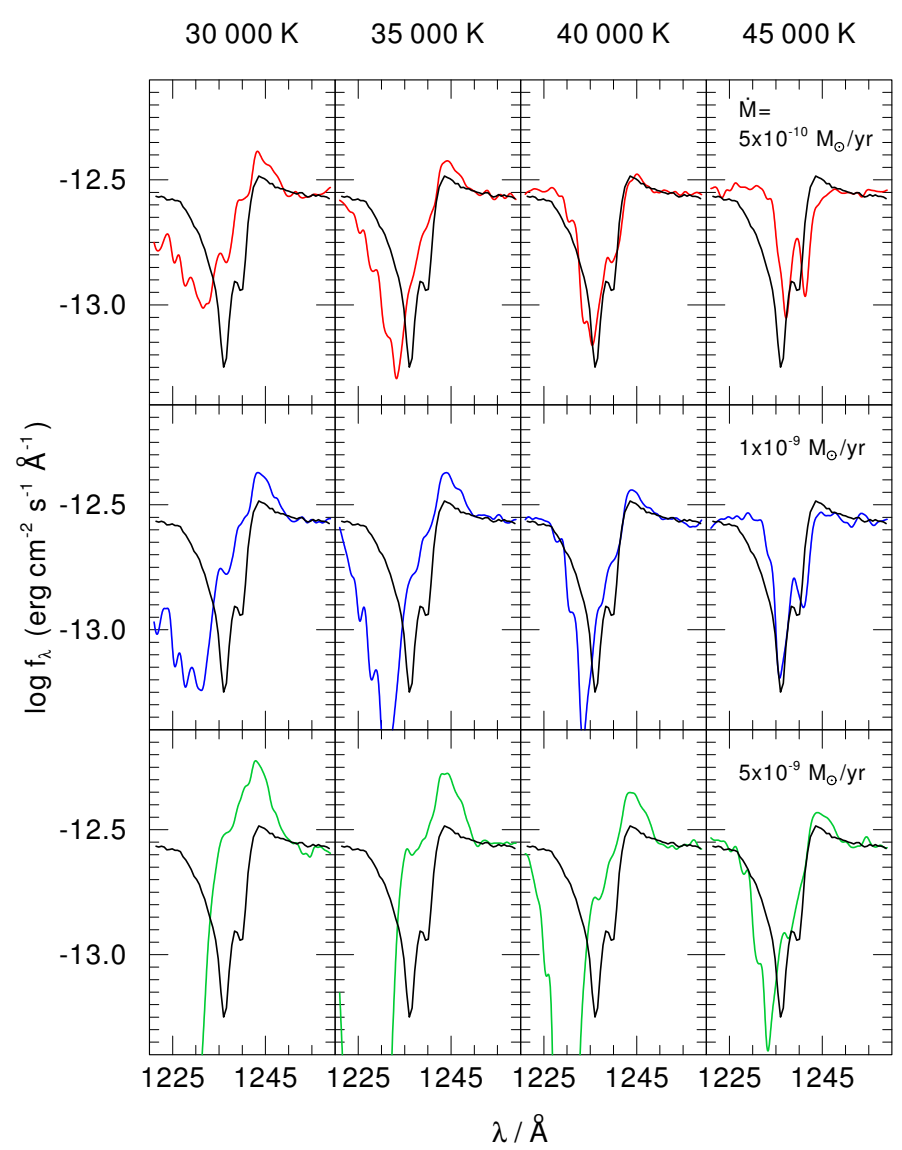

Fig. 14. Same as Fig. 12, but for $i=60^{\circ}$. 


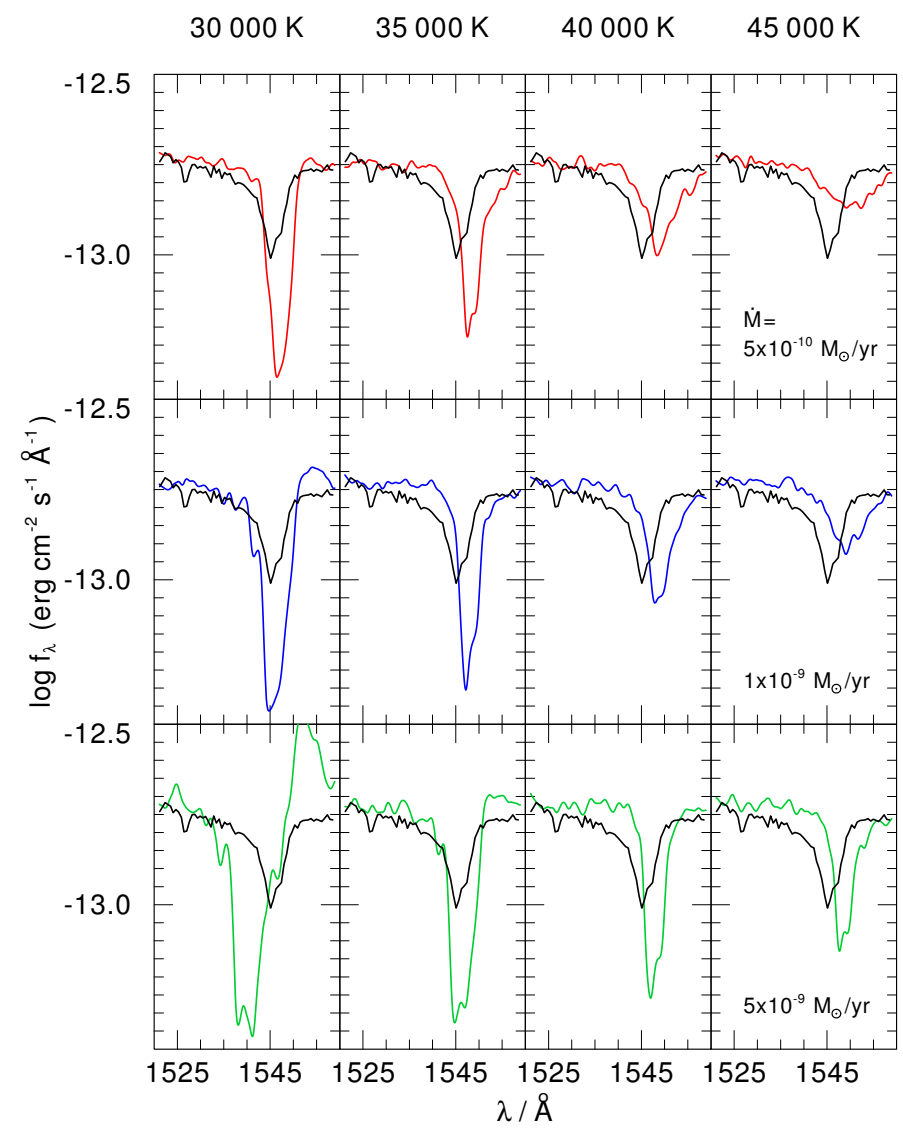

Fig. 15. Same as Fig. 13, but for $i=60^{\circ}$.

- Applying our WoMPaT code to model the helium "novalike" AM CVn has been successful in reproducing the prominent PCygni profiles, which our disk models lacked up to now. Comparing our SV model calculations to UV observations of $\mathrm{AMCVn}$, we found the best agreement for $\dot{M}_{\text {wind }}=5 \times 10^{-10} M_{\odot} \mathrm{yr}^{-1}$ and $T_{\text {wind }}=35000 \mathrm{~K}$ using the most probable literature value of $i=40^{\circ}$ for the system inclination. However, the $\mathrm{N}$ abundance had to be five times solar to model the line strength of the $\mathrm{N} v$ line at about $1240 \AA$.
We also used WoMPaT to model PCygni wind profiles in SS Cygni and ZCamelopardis (Kusterer 2008). We compared available observations with calculated models with different values for $\dot{M}_{\text {wind }}$ and $T_{\text {wind }}$ again using AcDc disk models as well as blackbody spectra to represent the accretion disk. Although first results are promising, more parameter studies have to be made to conclude with a definite answer regarding these values.

Furthermore, our results show the necessity of a variable temperature structure in the wind, such as Long \& Knigge (2002) or Noebauer et al. (2010) use in their models, to reproduce different wind lines with the same quality. This will be one of the first steps in the future development of our wind code.

Acknowledgements. This work was supported by DFG grants Fe 477/3-1 and We $1312 / 37-1$. We thank the bwGRiD project ${ }^{1}$ for the computational resources. We thank the referee for his/her constructive criticism that helped to improve the paper.

\section{References}

Abbott, D. C. 1980, ApJ, 242, 1183

Cassinelli, J. P. 1979, ARA\&A, 17, 275

Castor, J. I., Abbott, D. C., \& Klein, R. I. 1975, ApJ, 195, 157

Cordova, F. A., \& Mason, K. O. 1982, ApJ, 260, 716

Downes, R., Webbink, R. F., \& Shara, M. M. 1997, PASP, 109, 345

Feldmeier, A., \& Shlosman, I. 1999, ApJ, 526, 344

Feldmeier, A., Shlosman, I., \& Vitello, P. 1999, ApJ, 526, 357

Gräfener, G., \& Hamann, W.-R. 2005, A\&A, 432, 633

Harvey, D. A., Skillman, D. R., Kemp, J., et al. 1998, ApJ, 493, L105

Heap, S. R., Boggess, A., Holm, A., et al. 1978, Nature, 275, 385 Hillier, D. J., Lanz, T., Heap, S. R., et al. 2003, ApJ, 588, 1039

Knigge, C., Woods, J. A., \& Drew, J. E. 1995, MNRAS, 273, 225

Kusterer, D.-J. 2008, Ph.D. Thesis, University of Tübingen, Germany Long, K. S., \& Knigge, C. 2002, ApJ, 579, 725

Morales-Rueda, L., Marsh, T. R., Steeghs, D., et al. 2003, A\&A, 405, 249

Nagel, T., Dreizler, S., Rauch, T., \& Werner, K. 2004, A\&A, 428, 109

Nagel, T., Rauch, T., \& Werner, K. 2009, A\&A, 499, 773

Noebauer, U. M., Long, K. S., Sim, S. A., \& Knigge, C. 2010, ApJ, 719, 1932

Proga, D., Stone, J. M., \& Drew, J. E. 1998, MNRAS, 295, 595

Proga, D., Stone, J. M., \& Drew, J. E. 1999, MNRAS, 310, 476

Puebla, R. E., Diaz, M. P., Hillier, D. J., \& Hubeny, I. 2011, ApJ, 736, 17

Ramsay, G., Groot, P. J., Marsh, T., et al. 2006, A\&A, 457, 623

Roelofs, G. H. A., Groot, P. J., Benedict, G. F., et al. 2007, ApJ, 666, 1174

Shakura, N. I., \& Sunyaev, R. A. 1973, A\&A, 24, 337

Shlosman, I., \& Vitello, P. 1993, ApJ, 409, 372

Solheim, J.-E. 2010, PASP, 122, 1133

Wade, R. A., Eracleous, M., \& Flohic, H. M. L. G. 2007, AJ, 134, 1740
1 bwGRiD (http://www.bw-grid.de), member of the German D-Grid initiative, funded by the Ministry for Education and Research (Bundesministerium für Bildung und Forschung) and the Ministry for Science, Research and Arts Baden-Württemberg (Ministerium für Wissenschaft, Forschung und Kunst Baden-Württemberg). 